\title{
Recombination of Polaronic Electron-hole Pairs in Hematite Determined by Nuclear Quantum Tunneling
}

\author{
Yunyan Fan, Yumei Lin, Kelvin H. L. Zhang*, Ye Yang* \\ State Key Laboratory of Physical Chemistry of Solid Surfaces, College of Chemistry and \\ Chemical Engineering, Xiamen University, Xiamen 361005, China
}

\section{Experimental methods.}

\subsection{Fabrication of the hematite single crystal films.}

Pulsed laser deposition (PLD) method was employed to grow $\alpha-\mathrm{Fe}_{2} \mathrm{O}_{3}$ epitaxial single crystal films on $\mathrm{Al}_{2} \mathrm{O}_{3}$ (0001) substrates. The high-purity $\mathrm{Fe}_{2} \mathrm{O}_{3}$ powder (99.99\%, Alfa Aesar) sintered in air at temperature of $1250^{\circ} \mathrm{C}$ for 12 hours was used as the target material. The ablation of the target was carried out by using a $\operatorname{KrF}$ excimer laser $(\lambda=248 \mathrm{~nm})$ with pulse repetition rate of $5 \mathrm{~Hz}$. The energy density of the laser irradiation is of $1.2 \mathrm{~J} / \mathrm{cm}^{2}$. The ablation material was deposited on the $\mathrm{Al}_{2} \mathrm{O}_{3}$ (0001) substrate that was heated to the temperature of $550^{\circ} \mathrm{C}$ with an oxygen background pressure of 100 mTorr during the growth. The growth of the single crystal film was monitored by the in-situ reflection high-energy electron-diffraction (RHEED). The resulting film was cooled to room temperature under the same $\mathrm{O}_{2}$ background pressure. The thicknesses of the $\alpha-\mathrm{Fe}_{2} \mathrm{O}_{3}$ film used in this study is about $40 \mathrm{~nm}$. The crystal structure characterization and spectroscopic examination of the $\alpha-\mathrm{Fe}_{2} \mathrm{O}_{3}$ films grown under the same condition have been previously reported..$^{1-2}$

\subsection{Femtosecond transient absorption (TA) measurement.}

The TA setup is based on a Ti:sapphire amplifier (Coherent Astrella) that generates $1 \mathrm{KHz} 800 \mathrm{~nm}$ pulses with temporal width of $35 \mathrm{fs}$. The output pulse energy is around $7 \mathrm{~mJ}$. A branch of the fundamental beam ( $2.8 \mathrm{~mJ} /$ pulse) was introduced into an optical parametric amplifier (OPerA Solo, Coherent) to produce the pump pulses. For this study, the pump wavelength was tuned to $500 \mathrm{~nm}$. The $1 \mathrm{KHz}$ pump pulses were chopped at the frequency of $500 \mathrm{~Hz}$, and the pulse energy was attenuated to $193 \mathrm{~nJ} /$ pulse by neutral density filter wheels. Another branch of the fundamental beam ( $\sim .1 \mathrm{~mJ} /$ pulse) was attenuated and then focused 
into the sapphire crystal to generate the white light continuum (450-800 nm), which was used as the probe. The time delay between the pump and probe pulses was controlled by a motorized translation stage. Both pump and probe pulses were focused on the same position of the sample. The transmitted pump pulses were blocked. The transmitted probe pulses were sent into to a spectrometer. The sample was placed into the vacuum chamber of a liquid nitrogen cryostat (LNC-W, Lanhai Instrument).

At the sample position, the focal size of the pump was intentionally adjusted to be much greater than that of the probe. Thus, the excitation density of the probing area was nearly homogenous. The radii of pump and probe beams at the sample position were measured to be $\sim 450 \mu \mathrm{m}$ and $\sim 50 \mu \mathrm{m}$, respectively. The density of absorbed photons was determined according to the following equation,

$$
n=\frac{J_{\text {in }}}{h v} \cdot T_{w}\left(1-R_{s}-T_{s}\right) /\left(\pi r^{2} L\right)
$$

where $J_{\text {in }}$ was the pulse energy of the incident pump, $h v$ is the photon energy of the pump, $T_{w}$ was the transmittance of the chamber window $\left(T_{w}=93 \%\right), R_{S}$ was the reflectivity of the sample $\left(R_{S}=48 \%\right), T_{S}$ was the transmittance of the sample $\left(T_{S}=29 \%\right), r$ was the radius of pump beam and $L$ was the thickness of the film. The absorbed-photon density was then determined to be $3.3 \times 10^{18} \mathrm{~cm}^{-3}$,

\section{Supplementary Figures.}

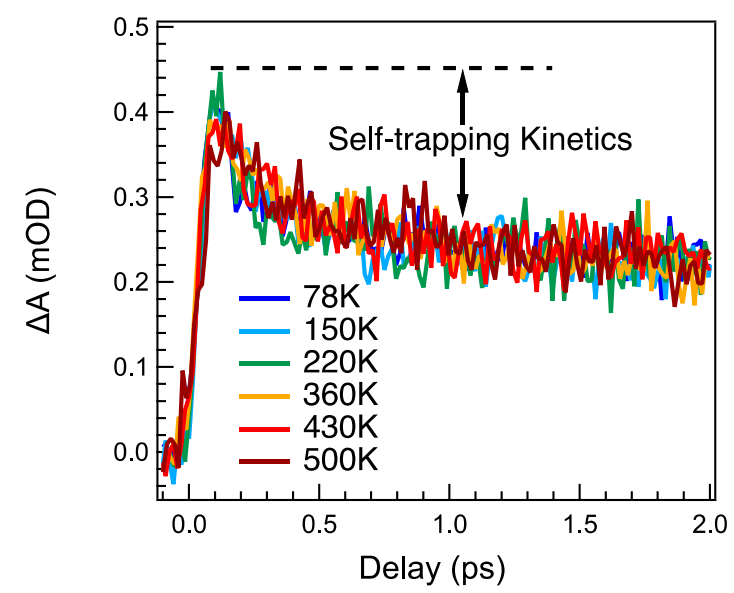

Figure S1. Self-trapping kinetics measured at different temperatures. The sub-ps ultrafast decay of the deep sub-bandgap PIA kinetics has been attributed to the self-trapping of free carriers. $^{2-3}$ The comparison of the self-trapping kinetics measured at different temperatures suggests that the self-trapping dynamics does not have clear temperature dependence. 

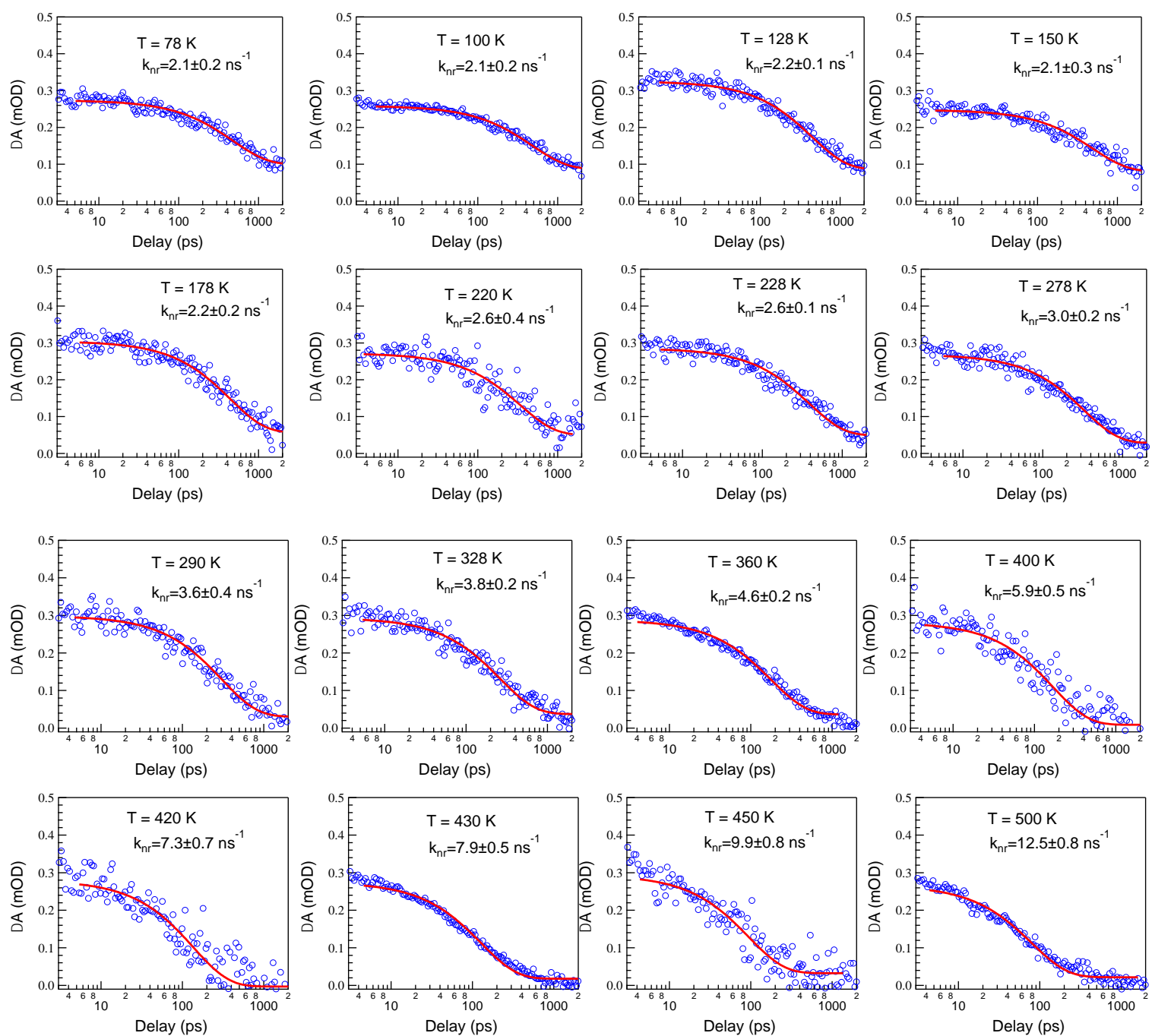

Figure S2. STE recombination kinetics and the corresponding single-exponential fitting curves for different temperatures. For most TA measurements, the fitting uncertainty of the STE recombination kinetics is smaller than the standard deviation of the multiple measurements, and then in this case the standard deviation is used as the error bar. For some data sets (e.g., $400 \mathrm{~K}, 420 \mathrm{~K}$ and 450K), the fitting uncertainty exceeds the standard deviation of different measurements, and then the fitting uncertainty is adopted as the error bar. 

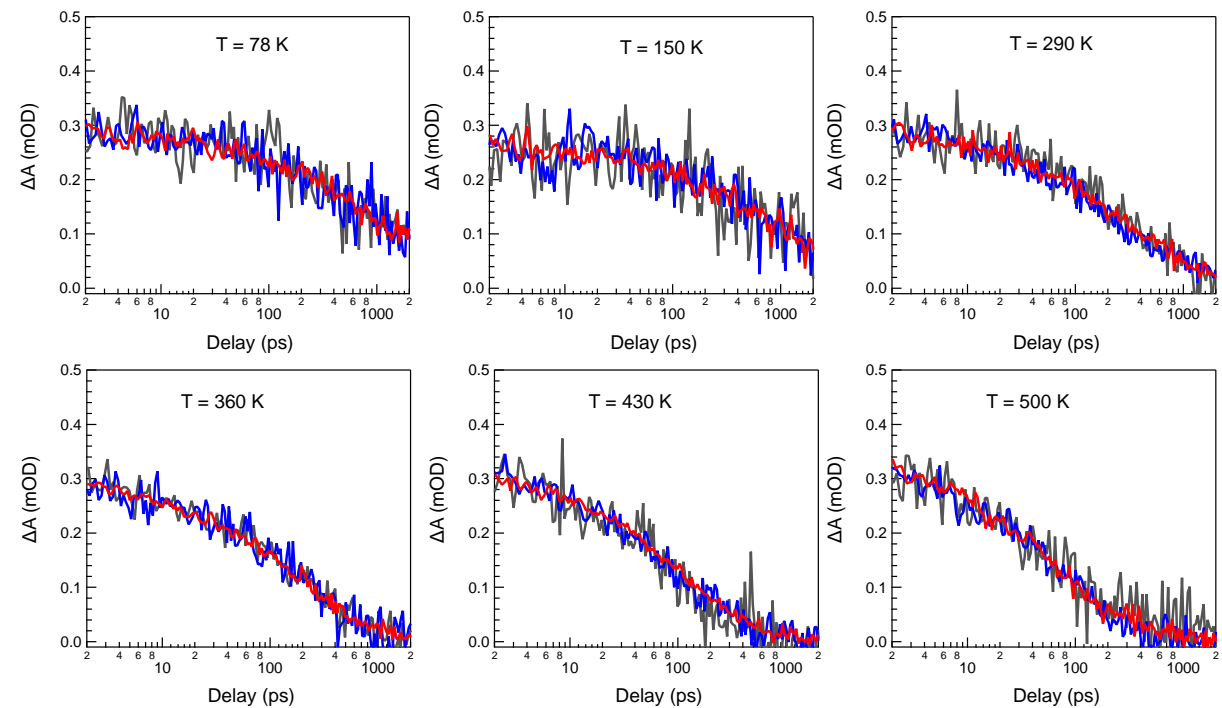

Figure S3. Comparison of three sets of STE recombination dynamics measured under similar conditions for different temperatures. The lower single-to-noise ratio is mainly caused by reduced number of averaged measurements. Because the pump intensity was not controlled to be exactly the same, the maximum signal amplitude varies from $\sim 0.2 \mathrm{mOD}$ to $\sim 0.3 \mathrm{mOD}$. We have demonstrated that the STE recombination dynamics is independent of the pump intensity under low excitation condition, ${ }^{2}$ thus the small variation of the pump intensity should not affect the recombination rate. The kinetics were normalized to the same initial amplitude for a better comparison of the decay trend.

1. Tian, C. M.; Li, W. W.; Lin, Y. M.; Yang, Z. Z.; Wang, L.; Du, Y. G.; Xiao, H. Y.; Qiao, L.; Zhang, J. Y.; Chen, L., et al. Electronic structure, optical properties, and photoelectrochemical activity of sn-doped fe2o3 thin films. J. Phys. Chem. C 2020, 124 (23), 12548-12558.

2. Liao, H.; Fan, Y.; Lin, Y.; Wang, K.; Li, R.; Chen, X.; Zhang, K. H. L.; Yang, Y. Microheterogeneous annihilation dynamics of self-trapped excitons in hematite single crystals. $J$ Phys Chem Lett 2020, 11 (18), 7867-7873.

3. Fan, Y.; Lin, Y.; Wang, K.; Zhang, K. H. L.; Yang, Y. Intrinsic polaronic photocarrier dynamics in hematite. Phys. Rev. B 2021, 103 (8), 085206. 